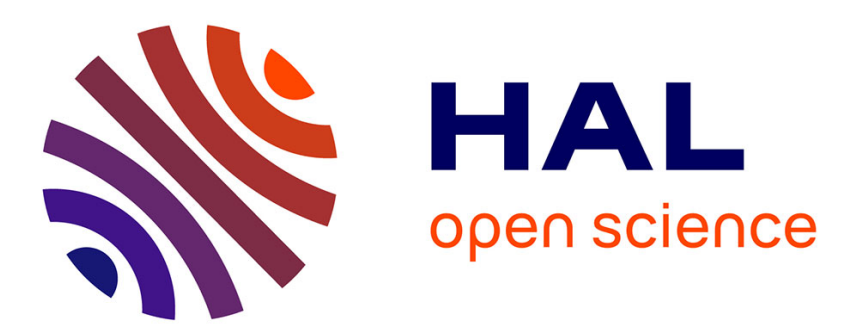

\title{
Determining the Maximal Singularity-free Circle or Sphere of Parallel Mechanisms Using Interval Analysis
}

Mohammad Hadi Farzaneh Kaloorazi, Mehdi Tale Masouleh, Stéphane Caro

\section{To cite this version:}

Mohammad Hadi Farzaneh Kaloorazi, Mehdi Tale Masouleh, Stéphane Caro. Determining the Maximal Singularity-free Circle or Sphere of Parallel Mechanisms Using Interval Analysis. Robotica, 2016, 34 (1), pp.135 -149. 10.1017/S0263574714001271 . hal-01084688

\section{HAL Id: hal-01084688 \\ https://hal.science/hal-01084688}

Submitted on 20 Nov 2014

HAL is a multi-disciplinary open access archive for the deposit and dissemination of scientific research documents, whether they are published or not. The documents may come from teaching and research institutions in France or abroad, or from public or private research centers.
L'archive ouverte pluridisciplinaire HAL, est destinée au dépôt et à la diffusion de documents scientifiques de niveau recherche, publiés ou non, émanant des établissements d'enseignement et de recherche français ou étrangers, des laboratoires publics ou privés. 


\title{
Determining the Maximal Singularity-free Circle or Sphere of Parallel Mechanisms Using Interval Analysis
}

\author{
Mohammad Hadi Farzaneh Kaloorazi ${ }^{1}$, Mehdi Tale Masouleh ${ }^{2}$ and Stéphane Caro ${ }^{3}$
}

\begin{abstract}
This paper proposes a systematic algorithm based on the interval analysis concept in order to obtain the maximal singularity-free circle or sphere within the workspace of parallel mechanisms. As case studies the 3-RPR planar and 6-UPS parallel mechanisms are considered to illustrate the relevance of the algorithm for $2 \mathrm{D}$ and $3 \mathrm{D}$ workspaces. To this end, the main algorithm is divided into four sub-algorithms, which eases the understanding of the main approach and leads to a more effective and robust algorithm to solve the problem. The first step is introduced to obtain the constant-orientation workspace and then the singularity locus. The main purpose is to obtain the maximal singularity-free workspace for an initial guess. Eventually, the general maximal singularity-free workspace is obtained. The main contribution of the paper is the proposition of a systematic algorithm to obtain the maximal singularity-free circle/sphere in the workspace of parallel mechanisms. The combination of the maximal singularity-free circle or sphere with the workspace analysis by taking into account the stroke of the actuators, as additional constraint to the latter problem, is considered. Moreover, the center point of the circle/sphere is not restrained to a prescribed point.
\end{abstract}

\section{NOMENCLATURE}

[.] An interval variable

$[\mathcal{W}],[\mathcal{S}]$ Lists of interval variables

$\left[\mathbf{b}_{\mathrm{C}}\right]$ A two dimensional interval variable, called current box

$\mathcal{D} \quad$ List of scalar variables, namely distance

$C_{f} \quad$ Final center point

CAD Computer Aided Design

CAM Computer Aided Machining

DOF Degree Of Freedom

EE End-Effector

FKP Forward Kinematic Problem

IKP Inverse Kinematic Problem

MSFC Maximal Singularity-Free Circle

MSFS Maximal Singularity-Free Sphere

P Prismatic joint

PPM Planar Parallel Mechanisms

$\mathrm{R} \quad$ Revolute joint

$\mathrm{S} \quad$ Spherical joint, Consists of 3 perpendicular revolute joint

U Universal joint, Consists of 2 perpendicular revolute joint

\footnotetext{
${ }^{1}$ University of Tehran, Faculty of New Sciences and Technologies, North Kargar, Tehran, Iran, mhfarzane@ut.ac.ir

${ }^{2}$ University of Tehran, Faculty of New Sciences and Technologies, North Kargar, Tehran, Iran, m.t.masouleh@ut.ac.ir

${ }^{3}$ IRCCyN/CNRS, UMR 6597, 1 rue de la Noë, 44321 Nantes, France, stephane.carodirccyn.ec-nantes.fr
}

\section{INTRODUCTION}

Due to the remarkable properties of Parallel Mechanisms (PMs) [1]-[5], such as higher payload to weight ratio and higher accuracy, they are now the state-of-the-art of a wide range of commercial context, such as the Gough-Stewart platform for flight simulator and Delta robots for pick and place applications. More details on the true origins of PMs is elaborated in [6]. However, there are some major deterrents to widespread PMs in the industrial contexts, including, among others, mathematics complexities in analyzing their kinematic properties, the extensive presence of uncontrollable configurations, referred to as singularities [7], within their restricted workspace.

Since the limited workspace of PMs is coupled with singularities, in the design stage of a PM the workspace analysis and singularity analysis are of paramount importance which they should be analyzed in such a way that leads to a PM with singularity-free workspace [5], [8], [9]. In singular configuration a PM loses its inherent rigidity [10], [11] and mathematically can be related to the singularity of some Jacobian matrices [7], [12]. In fact, Jacobian matrices provide the mapping between the joint rates and the Cartesian velocities of the mechanism which are arising from the firstorder kinematic properties of the mechanism, i.e., equations coming from the differentiation of the IKP with respect to time. In the literature, the singularities of PMs are classified upon different perspectives [13], and in this paper the one proposed in [7] is used whose perspective is perhaps the closest in spirit to the logic of this paper. Directly from [7], the singularities of PMs fall into three types: (1) Type I, inverse kinematic singularity, Type II direct kinematic singularity and Type III a combination of Type I and II. Due to the high importance of type II singularity, the latter is considered for the purpose of this paper.

There has been an extensive study conducted on the singularity-free workspace of PMs where most of them are based on complicated numerical approaches and entail some limits. They could not be extended to many kind of parallel mechanisms and they are proposed for a prescribed center point. It is of paramount importance to study the singularityfree workspace of a PM before going into the design stage and this can be exemplified by the number of the papers published on this issue. Bonev et al. [14] conducted an exhaustive study on the singularity locus of planar 3-degreeof-freedom (DOF) PMs by resorting to screw theory. In [15], a method based on the geometrical parameters is proposed for which the singularity-free workspace of a three-legged 
PM is obtained. Li et al. [16], by using the fact that the problem of maximum singularity-free circle of 3-DOF PMs can be expressed mathematically as an optimization problem accompanied with a constraint resorted to the Lagrangian multipliers to solve the problem and maximal singularity-free zone which is a circle for a prescribed point was obtained. Jiang and Gosselin in [17]-[19] proposed some numerical recipes in order to find the singularity-free workspace of 3-DOF PMs. Recently, in [20], upon resorting to particle swarm optimization the maximum singularity-free circle of a 3-DOF PM was obtained for a prescribed center point. Moreover, in [11], the problem of closeness to singularity is addressed by formulating the question in terms of constrained optimization problem.

The motivation behind choosing the circle/sphere is twofold: the workspace could be replaced by a convex shape, i.e., a circle/sphere, which would be of great importance in kinematic properties optimization, and (2) it could be used for the problem of dimensional synthesis, i.e., the design parameters, such as the base and end-effector size and leg length of the PM, are of interest by prescribing the circle/sphere. In practice, usually it is desired to have a symmetric shape of the workspace. The singularity-free workspace is interesting for trajectory planning [21]. A method was presented in [22] to determine whether there is a singularity in a given region defined in the workspace. The answer is definite and can be used to identify the singularity-free zones inside the workspace. The singularity-free workspace of planar parallel manipulators with prismatic joints was addressed in [23]. Both the base and the platform of the used manipulator are collinear. The singularity problem of planar 3-RPR parallel mechanisms was studied in [16], in which a circle for a prescribed point was obtained. The singularity problem of the general Gough-Stewart platform was addressed in [24], where a procedure was presented to determine a maximal singularity-free zone which is a sphere around a point of interest $P_{0}$ for a prescribed orientation. This method was also extended to the six-dimensional workspace.

This paper aims at establishing a systematic approach based on interval analysis [25] to obtain the Maximal Singularity-Free Circle (MSFC)—in the case of 3DOF planar PMs (PPM) - and Maximal Singularity-Free Sphere (MSFS) -in the case of 6-DOF Gough-Stewart platforms-for which the center of the circle is not given. Moreover, the boundaries of the workspace is also taken into account in the analysis. In the literature, some analytical approaches are introduced, i.e. Lagrangian equations, which are hard to be extended to other problems. The aforementioned points distinguishes this work from others reported in the literature [8], [15], [16], [20].

The proposed algorithm does not depend on the structure of the mechanism and is applicable to almost every mechanisms. But, resorting to interval analysis, in the case of complicated mechanisms having a high degree singularity expression, it may lead to a very time consuming and inefficient process to obtained the aforementioned workspace. Use of consistency techniques may solve such a problem [26].

The remainder of the paper is organized as follows. First, the general concept of interval analysis is presented. In Section 3, the proposed algorithm is fully explained and four pseudo-codes are provided for a better understanding of the problem. Moreover, some results are given which certify to show the effectiveness of the proposed algorithms. It noteworthy to say that the kinematic modelling of some case studies are addressed in the last section, i.e., the so called 6-UPS PM and 3-RPR Planar Parallel Manipulator (PPM).

\section{INTERVAL ANALYSIS AND MATHEMATICAL} FRAMEWORK

Several people independently had the idea bounding rounding errors by computing with intervals; e.g. Dwyer (1951) [27], Sunuga (1958) [28], Warmus (1956) [29] and Wilkinson (1980) [30]. However, interval mathematics can be said to have begun with the appearance of R. E. Moore's book "Interval Analysis" in 1966 [31]. Moore's book transformed this simple idea into a viable tool for error analysis.Instead of merely treating rounding errors, Moore extended the use of interval analysis to bound the effect of errors from all sources, including approximation and errors in data [32]. In the literature, interval analysis is regarded as a powerful numerical method to solve a wide range of problems such as, among others, circumventing round-off errors [33], solving system of equations, optimization problem [32] and proper workspace presentation, etc. [32], [34]-[36]. Furthermore, interval analysis provides an interactive visualization in the progress of calculation which is a definite asset in $2 \mathrm{D}$ and $3 \mathrm{D}$ representations of manipulator workspaces. Recently, upon revealing some remarkable features of interval analysis, such as finding the solution of a problem within some finite domain and taking into account the numerical computer round-off errors, it has stimulated the interests of many researchers in robotic community to deal with complicated problems such as IKP, FKP, calibration and the determination of the singularity-free workspace of parallel manipulator, the main concern of this paper.

Here is a list of advantages of using interval analysis instead of evolutionary approaches such as genetic algorithm. In the evolutionary techniques the chance of being trapped in a local optima is highly depended on the initial population and initial search space. However, in the case of interval analysis, the only parameter to obtain the global optima is choosing the proper search space. In order to compute the maximum singularity-free workspace of PMs and other kinematic properties [34], [35], interval analysis entails following advantages: (1) Contrary to other tools, which would result in a lengthy computation process and may converge to a local optimum, interval analysis is not a black box, since it requires combination of heuristics and numerical concepts 
to be effective; (2) It allows us to find all the solutions with inequalities within a given search space [33], [37]; (3) For two and three-dimensional problems, it leads to see the evolution of the solutions and to monitor the procedure in order to have better insight to the problem; (4) It allows us to consider uncertainties in the model of the robot.

Interval analysis, is a branch of mathematics that basically works with closed intervals instead of accurate numbers. An interval $[x]$ is a set of real numbers between two bounds and can be represented as:

$$
[x]=[\underline{x}, \bar{x}]=\{x \in \mathbb{R} \mid \underline{x} \leq x \leq \bar{x}\},(\underline{x} \leq \bar{x})
$$

where $\underline{x}$ and $\bar{x}$ are lower bound and upper bound, respectively. All mathematical operations such as addition and multiplication can be performed on intervals. For instance [25]:

$$
\begin{gathered}
{[x]+[y]=[\underline{x}, \bar{x}]+[\underline{y}, \bar{y}]=[\underline{x}+\underline{y}, \bar{x}+\bar{y}],} \\
{[x][y]=[\min (S), \max (S)], \quad S=\{\underline{x} \underline{y}, \underline{x} \bar{y}, \bar{x} \underline{y}, \overline{x y}\} .}
\end{gathered}
$$

Moreover, a function of real numbers such as $f(x)$ can be evaluated as an interval from a given interval, $[x]$, which results in an interval $[f]=f([x])$. For example for a monotonic function like $f(x)=x^{3}$ :

$$
[f]=f([x])=[f(\underline{x}), f(\bar{x})]=\left[\underline{x}^{3}, \bar{x}^{3}\right] .
$$

The whole concept of interval analysis is based on bisecting a box (or a hyper-box in higher dimensional space), called branch \& prune approach [35], upon considering some well-defined algebra on intervals, in such a way that the latter box will converge toward the desired solution. In this paper, we are taking a step back from what it has been done up to now in the the literature. In fact, more emphasis is placed on how one can define a procedure to evolve the first box, which is chosen arbitrary by the user, to generate new ones to converge to the desired solution. In short, as it will be explained in an upcoming section, upon blending some classical concepts of numerical analysis with interval analysis, some obstacles to obtain the singularityfree workspace can be eliminated.

\section{Obtaining the Workspace, Singularity-FreE ZONE AND MSFC/MSFS FOR A GIVEN BOX AND Prescribed ORIENTATION OF THE MOVING Platform}

In this section, four algorithms, denoted as Algorithm 1, 2, 3 and 4 are proposed. The first three algorithms are for a given orientation of the moving platform and applicable for a prescribed box, called current box $\left[\mathbf{b}_{\mathrm{C}}\right]$ which is defined by the user. In other words, in the aforementioned algorithms, there is no evolution on $\left[\mathbf{b}_{\mathrm{C}}\right]$ and no new boxes are generated outside of this box (creating boxes inside $\left[\mathbf{b}_{\mathbf{C}}\right]$ is an inherent concept of interval analysis) and all the results are only valid for $\left[\mathbf{b}_{\mathbf{c}}\right]$. It should be noted that the result of Algorithm 3 in practice is similar to what has been done in [16], [20]. Therefore, in this paper, in order to circumvent this problem, Algorithm 3 is improved, called 4, in such a way that based

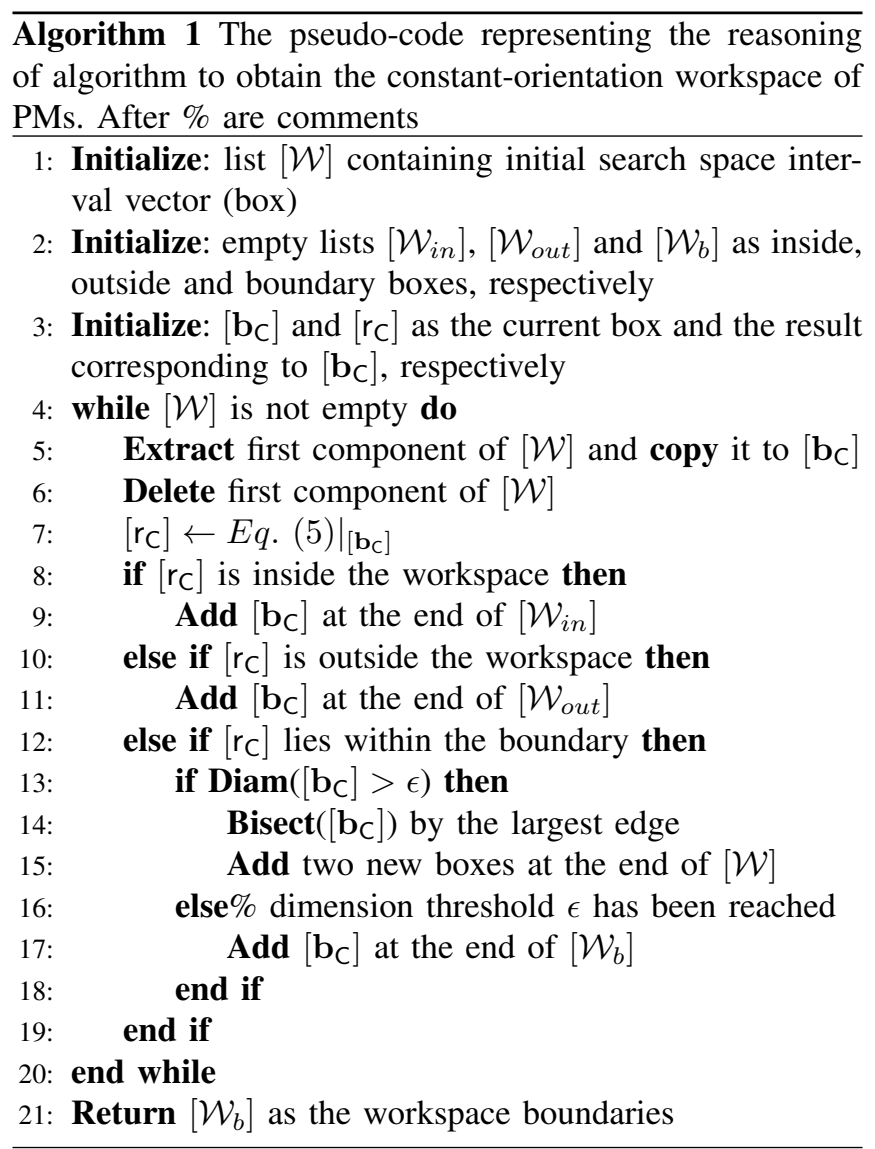

on some imposed rules new boxes are generated, which conduct us to the optimum solution for the MSFC/MSFS of the workspace as a whole. For the sake of clarity, lists are represented in Calligraphic $(\mathcal{C})$.

The proposed algorithms are represented in what follows. Note that for the sake of simplicity all procedures are explained for the 3-RPR planar PM, its constant-orientation is represented in $2 \mathrm{D}$. Then, the procedure can be readily developed to higher DOF PMs and later on, results are represented for 6-UPS PM.

\section{A. The Constant-orientation Workspace (Algorithm 1)}

The constant-orientation workspace of a PM, is the set of all feasible points for which the EE can reach for a prescribed orientation. Several methods are reported in the literature [38] [10] which range from numerical approaches to geometrical approach (such as the usage of CAD software [39]). It should be noted that several approaches based on interval analysis are proposed to solve the constantorientation workspace of PMs and in this paper, following the same reasoning as the one proposed in [10], an algorithm, represented in Algorithm 1 and denoted as A, is proposed. The problem of obtaining the constant-orientation workspace consists in solving the IKP, Eq. (9), for given $\rho_{\min }$ and $\rho_{\max }$, characterizing the stroke of the actuators, for a given orientation of the moving platform. Thus the problem reduces to solve inequalities as follows for $i=1,2,3$ for a 3-RPR PM and $i=1, \cdots, 6$ for a 6 -UPS PM in order to obtain the set 
of feasible points $(x, y)$ :

$$
\begin{aligned}
& \left(x_{A i}-x_{B i}\right)^{2}+\left(y_{A i}-y_{B i}\right)^{2} \leq \rho_{\max }^{2} \\
& \left(x_{A i}-x_{B i}\right)^{2}+\left(y_{A i}-y_{B i}\right)^{2} \geq \rho_{\min }^{2}
\end{aligned}
$$

Algorithm 1 shows a pseudo-code which solves the above system of inequalities in order to determine the constantorientation workspace. It should be noted that Algorithm 1 results in three types of lists which are the input data for the computation of singularity-free workspace for Algorithm 3 (1) the inner area of the workspace $\left(\left[\mathcal{W}_{\text {in }}\right]\right)(2)$ the outer area of the workspace $\left(\left[\mathcal{W}_{\text {out }}\right]\right)$ and (3) the boundaries of the workspace $\left(\left[\mathcal{W}_{b}\right]\right)$. If box $\left[\mathbf{b}_{C}\right]$ satisfies Eq. (5) for all legs simultaneously, then $\left[\mathbf{b}_{C}\right]$ will be inside the workspace and will be added to $\left[\mathcal{W}_{\text {in }}\right]$. If $\left[\mathbf{b}_{\mathrm{C}}\right]$ does not satisfy Eq. (5) even for one leg, it will be a part of $\left[\mathcal{W}_{\text {out }}\right]$, otherwise it will be added at the end of $[\mathcal{W}]$. As it can be observed from Algorithm 1, the algorithm continues the procedure until the dimension of $\left[\mathbf{b}_{C}\right]$ is higher than a given threshold $\epsilon$ which stands for the accuracy of the algorithm. Once the desired accuracy is reached, the remaining boxes will be considered as $\left[\mathcal{W}_{\mathrm{b}}\right]$.

For 3-RPR PPM the $\left[\mathbf{b}_{\mathrm{C}}\right]$ is a 2-dimensional box in $x y$ plane. If only for one leg, $\left[\mathbf{b}_{C}\right]$ is applied to Eq. (5), then this equation is converted to an interval function or equation. Therefore, the result of such a function is a interval, $\left[r_{C}\right]$.

$$
\left[\mathrm{r}_{\mathrm{C}}\right]=\left(x_{A i}-\left[\mathrm{b}_{\mathrm{C}_{x}}\right]\right)^{2}+\left(y_{A i}-\left[\mathrm{b}_{\mathrm{C}}\right]_{y}\right)^{2}
$$

If the upper bound of $\left[r_{\mathrm{C}}\right]$, called $\overline{r_{\mathrm{C}}}$, and lower bound of $\left[\mathrm{r}_{\mathrm{C}}\right], \underline{\mathrm{r}_{\mathrm{C}}}$, both are within the range of $\rho_{\min }^{2}<\cdot<\rho_{\max }^{2}$, then it can be deduced that $\left[\mathbf{b}_{\mathrm{C}}\right]$ satisfies the mechanical strokes for the corresponding leg. By the same token, $\left[\mathbf{b}_{C}\right]$ must satisfy all legs mechanical strokes.

\section{B. The Constant-Orientation Singularity locus (Algorithm 2)}

The reasoning applied for Algorithm 2 in order to obtain the inner area $\left(\left[\mathcal{S}_{\text {in }}\right]\right)$, the outer area $\left(\left[\mathcal{S}_{\text {out }}\right]\right)$ and the boundary $\left(\left[\mathcal{S}_{b}\right]\right)$ of the singularity is to the majority of intents and purposes the same as Algorithm 1 for the constant-orientation workspace and there is a slight difference to illustrate the algorithm in another viewpoint. In this case, Eq. (13), representing the singularity locus, should be considered. The pseudo-code of this section is represented in Algorithm 2. The differences between Algorithm 1 and Algorithm 2: if the result of substitution of $\left[\mathbf{b}_{C}\right]$ into Eq. (13) is negative, then it will be added to $\left[\mathcal{S}_{\text {in }}\right]$, otherwise it is completely positive and it will be a part of $\left[\mathcal{S}_{\text {out }}\right]$. In the case the box $\left[\mathrm{r}_{\mathrm{C}}\right]$ contains zero, it will be added at the end of $[\mathcal{S}]$. Algorithm 2 pursues the concept of interval analysis on the box $\left[\mathbf{b}_{\mathrm{C}}\right]$, defined latter, until the desired accuracy $\epsilon$ is reached. After reaching $\epsilon$, the remaining boxes are considered as $\left[\mathcal{S}_{\mathrm{b}}\right]$.

\section{The Constant-Orientation MSFC/MSFS for a Given Ini- tial Box (Algorithm 3)}

From Algorithms 1 and 2, having in hand the boundaries of the workspace and the singularity locus in an interval form, Algorithm 3, computes the optimum center and radius of the MSFC/MSFS for a given box, called initial guess box

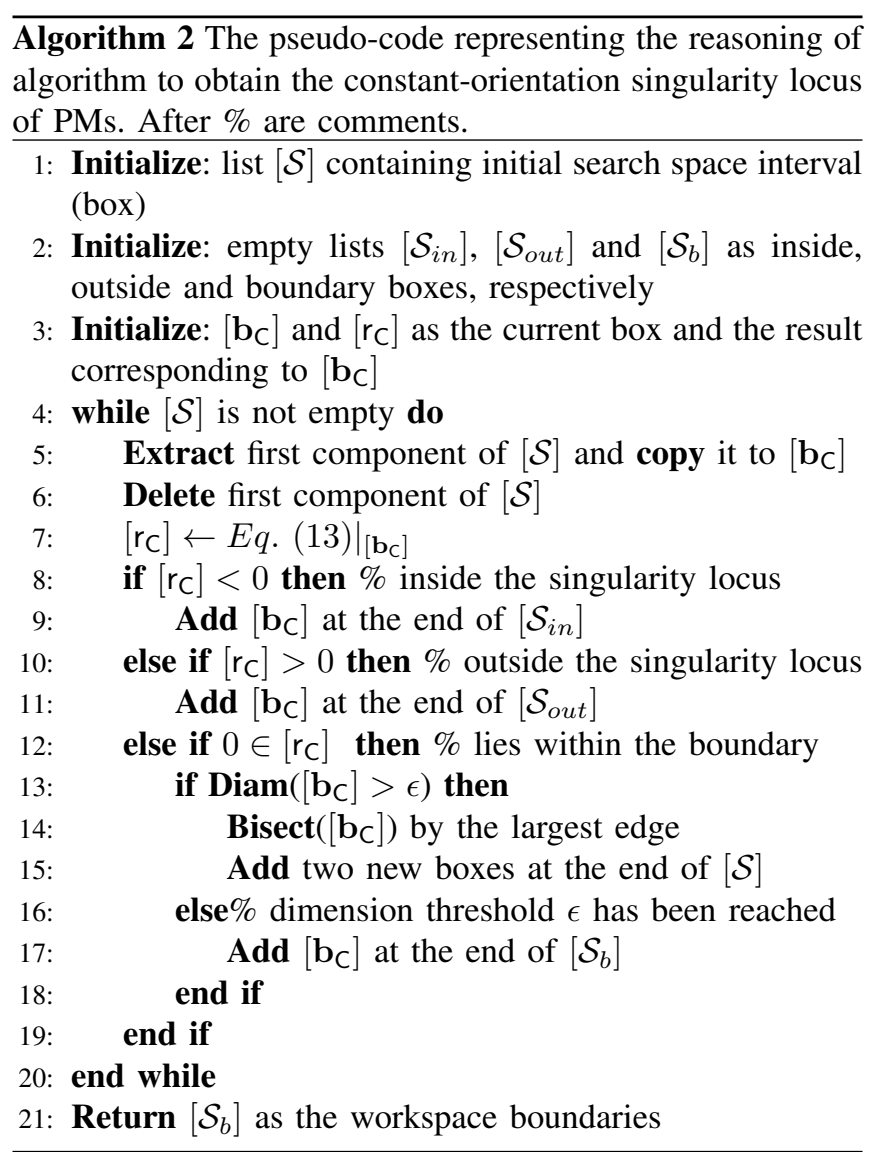

and denoted as $\left[\mathbf{b}_{0}\right]$. As mentioned previously, Algorithm 3 works only on the initial box $\left[\mathbf{b}_{0}\right]$, chosen arbitrary by the user, and, thus it may not contain the optimum center of the MSFC/MSFS. The algorithm starts first to substitute $\left[\mathbf{b}_{0}\right]$ into $\left[\mathbf{b}_{\mathrm{C}}\right]$. Then the concept of interval analysis is applied to $\left[\mathbf{b}_{\mathrm{C}}\right]$, meaning that two sub boxes, namely $\left[\mathbf{b}_{\mathrm{C} 1}\right]$ and $\left[\mathbf{b}_{\mathrm{C} 2}\right]$, are created by bisecting $\left[\mathbf{b}_{\mathrm{C}}\right]$. Then, the algorithm calculates the distances of the two new boxes obtained latter and consider the one being farther from the boundaries of the constantorientation workspace and the singularity locus which are listed in $[\mathcal{B}]$ (This part is done on the basis of the data provided by Algorithms 1 and 2).

If the desired accuracy, represented by $\epsilon$, is achieved then the procedure will end, otherwise the algorithm will substitute the corresponding box into $\left[\mathbf{b}_{\mathbf{C}}\right]$ and the procedure will continue. While it reaches the desired accuracy, the minimum value of $\mathcal{D}_{C i}, i=1,2$, gives the radius. Using the lower bound of $\mathcal{D}$ leads to ascertain that the obtained radius does not intersect the singularity locus. Then by a proper rounding, the center of the last interval is the center of the MSFC/MSFS for the current box $\left[\mathbf{b}_{0}\right]$ and the last radius derived form $\mathcal{D}$ is the radius of the $\mathrm{MSFC}$.

In Algorithm 3, if the initial box, $\left[\mathbf{b}_{0}\right]$ does not contain the center point of the MSFC/MSFS for the whole workspace, once the procedure reaches the accuracy of $\epsilon$, then the obtained center point is close to one edge of $\left[\mathbf{b}_{0}\right]$. Here, the definition of closeness is: the distance of the obtained 


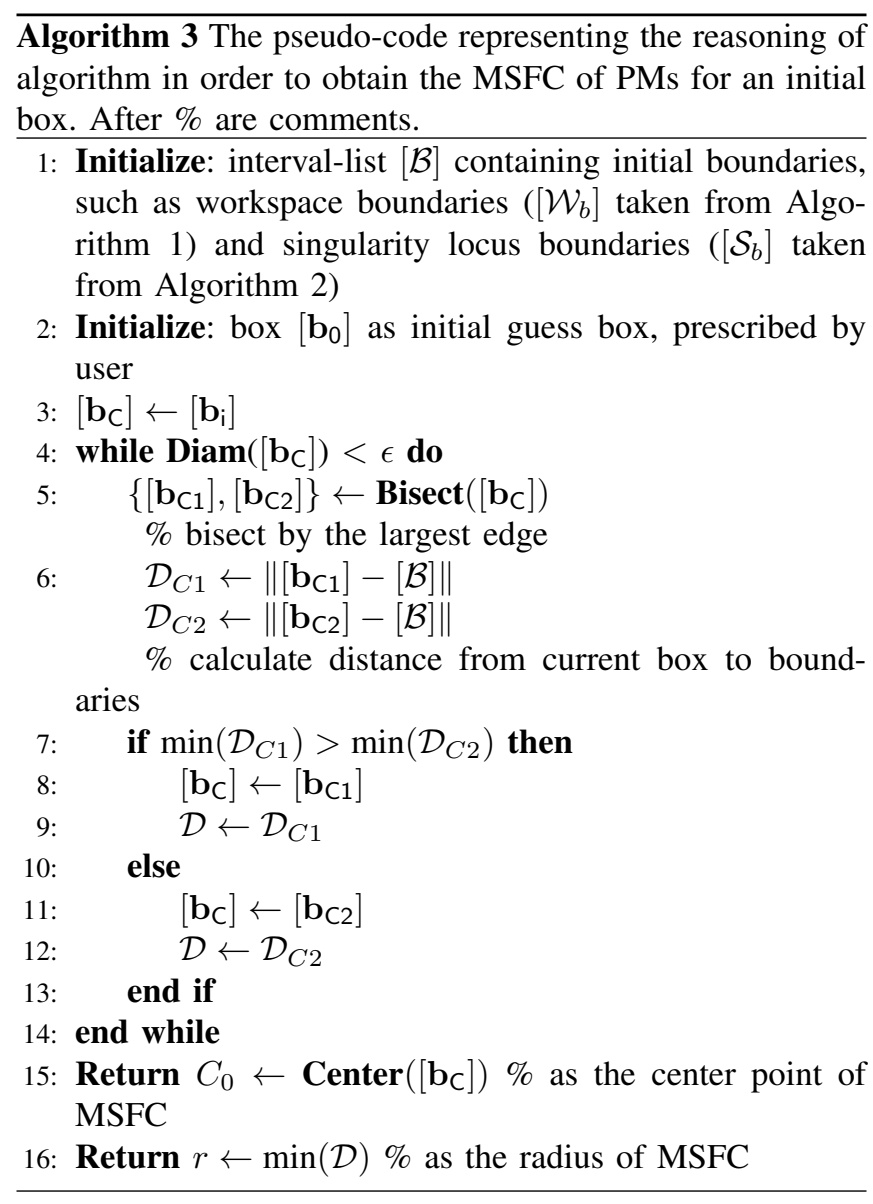

center point is lower than $\epsilon$. The next section will represent an approach, referred to as Improved MSFC/MSFS, which circumvent the latter problem and find the MSFC/MSFS for the workspace as a whole whether or not initial guess box contain the center of optimum circle/sphere.

\section{The Algorithm to Obtain the Improved MSFC/MSFS, Independent of the Initial Guess Box (Algorithm 4)}

Reaching this step, to the end of circumventing some shortcomings of Algorithm 3, new features are given to Algorithm 3 in order to present a new algorithm which guarantees that the obtained MSFC/MSFS is an optimum solution for the workspace as a whole. This algorithm, numbered 4, can generate new boxes from the initial box $\left[\mathbf{b}_{0}\right]$ defined by user in order to converge to the optimum MSFC/MSFS for the entire workspace.

Assume that $\left[\mathbf{b}_{0}\right]$ is an initial box for which Algorithm 3 is run. Then in the case that the obtained optimum center point corresponding to $\left[\mathbf{b}_{0}\right]$ is close to its edges, Algorithm 4 generates a new box, called $\left[\mathbf{b}_{1}\right]$, for which the same reasoning as Algorithm 3 is applied for. Algorithm 4 pursues to generate new boxes until the obtained center point would not be close to the edges of the box under study. The closeness to the edges of the box under study is based on a given criterion, such as $\epsilon$ defined previously.

In other words, when the obtained center point from Algorithm 3 is close to the edges of $\left[\mathbf{b}_{0}\right]$, one can predict

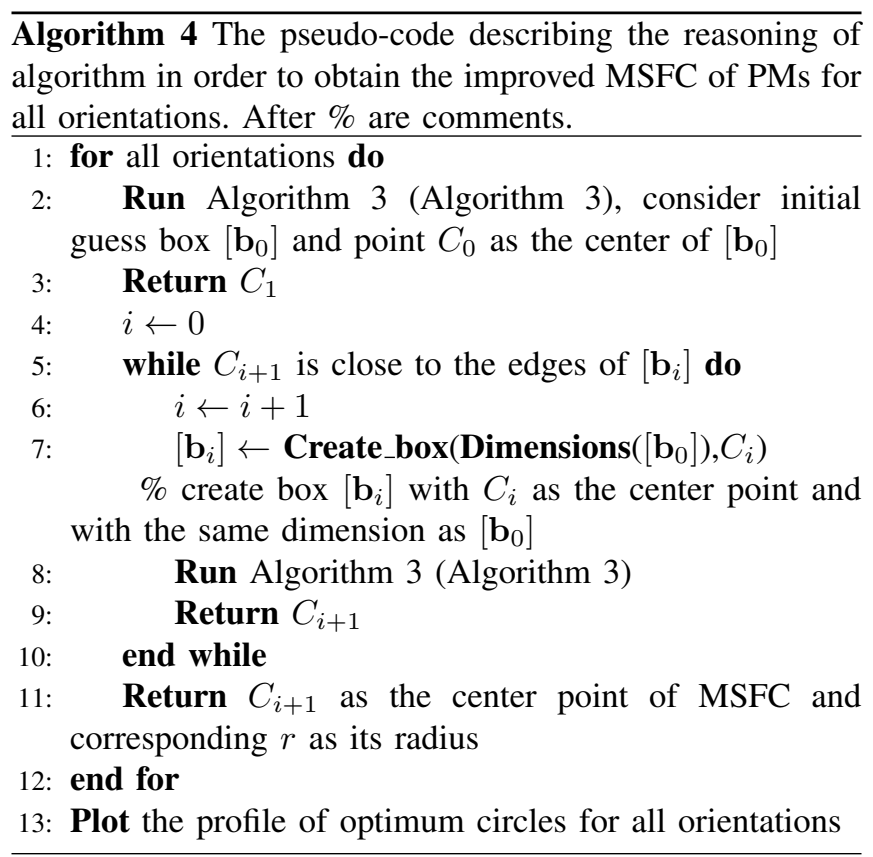

TABLE I

GEOMETRIC PARAMETERS OF A 3- $R \underline{P} R$ PM (ALL LENGTHS ARE GIVEN IN $\mathrm{mm}$ ).

\begin{tabular}{ccccccc}
\hline \hline$i$ & $B_{i x}$ & $B_{i y}$ & $\rho_{\min }$ & $\rho_{\max }$ & $P_{i x}^{\prime}$ & $P_{i y}^{\prime}$ \\
\hline 1 & 3.78 & 4.34 & 0.5 & 5 & -4.83 & -3.19 \\
2 & 34.47 & -3.78 & 0.5 & 5 & 12.04 & -3.19 \\
3 & 16.23 & 34.76 & 0.5 & 5 & 8.23 & 12.09 \\
\hline \hline
\end{tabular}

the direction for which the center point tends to approach toward its optimum value leading to the MSFC/MSFS of the workspace as a whole. Using this point as the center of a new box, called $\left[\mathbf{b}_{1}\right]$, which has the same size as $\left[\mathbf{b}_{0}\right]$, the Algorithm 4 can be used. The improved algorithm continues until the obtained center point would not be close to the edges of the box, $\left[\mathbf{b}_{i}\right], i=1,2, \cdots, n$, under study. The above algorithm is run for a given orientation of the moving platform and can be repeated for a range of moving platform orientations.

\section{RESUlTS}

\section{A. Results Obtained for 3-Rㅛ R Planar Parallel Mechanism}

In order to have a better insight into the reasoning of all algorithms proposed in this paper, this section is devoted to the analysis of the obtained solutions from Algorithms 1, 2, 3 and 4 for MSFC of a 3-RPR with design parameters as given in Table I.

Figure 1(a) represents the constant-orientation workspace, obtained from Algorithm 1, the singularity - a hyperbola in this case-obtained from Algorithm 2, for $\phi=0$. Then, in order to find the MSFC, an initial box $\left[\mathbf{b}_{0}\right]$ is selected for which the Algorithm 4 is applied. It should be noted that Algorithm 3 is inside Algorithm 4 and will be applied for each box generated by Algorithm 4, including the initial box $\left[\mathbf{b}_{0}\right]$. As it can be observed from Fig. 1(a), Algorithm 4 generates a new box, $\left[\mathbf{b}_{1}\right]$, since upon applying Algorithm 3 


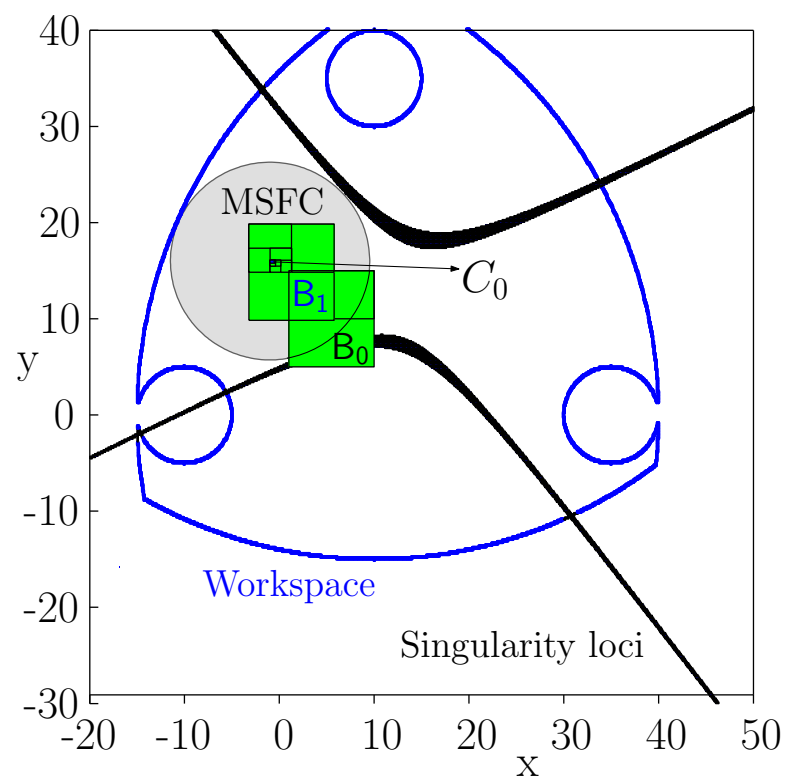

(a) $\phi=0$

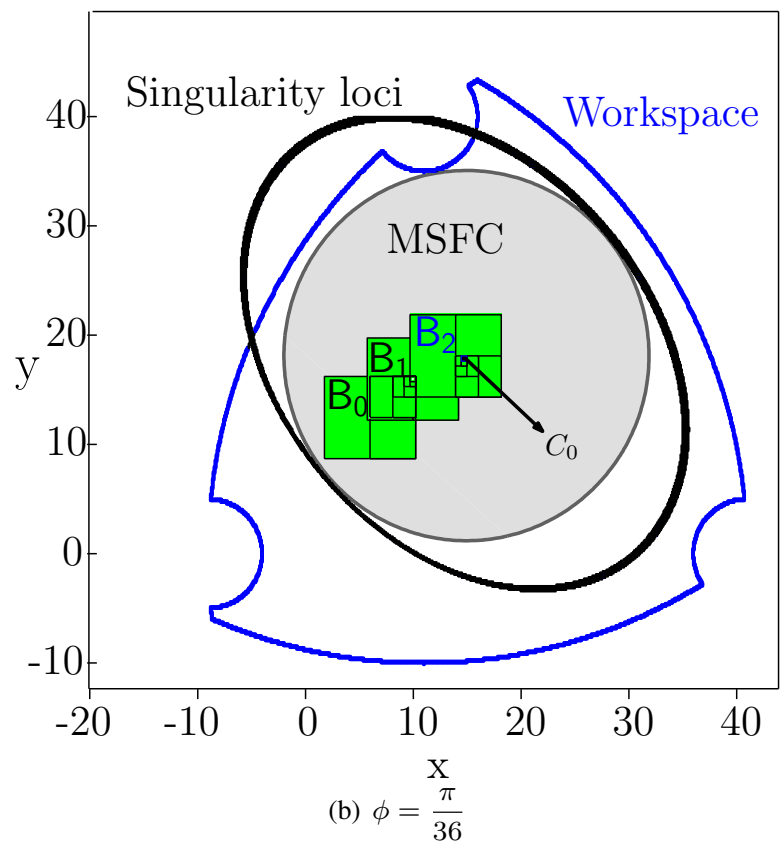

Fig. 1. The results for the workspace, singularity locus and MSFC for two different orientations of the moving platform in which new boxes are generated from $\mathrm{B}_{0}$ in order to find the center $C_{0}$ of the optimum center of the circle. For the sake of clarity and in order to not overload the figure some lines corresponding to small boxes produced by interval analysis are omitted.

into $\left[\mathbf{b}_{0}\right]$, the obtained center point lies on the boundary of $\left[\mathbf{b}_{0}\right]$. Finally, by applying Algorithm 3 on $\left[\mathbf{b}_{1}\right]$ the obtained center point is not on the boundary of $\left[\mathbf{b}_{1}\right]$ and consequently the obtained center point, $C_{f}$, is the optimum one for the MSFC and the radius can be readily obtained using the distance formula of two intervals.

Figures 1(b) represents a situation for which $\phi=\frac{\pi}{36}$ where, as it can be observed, the singularity locus is an ellipse. It can readily concluded that for the initial box, Algorithm 4 converges to the optimum MSFC by generating

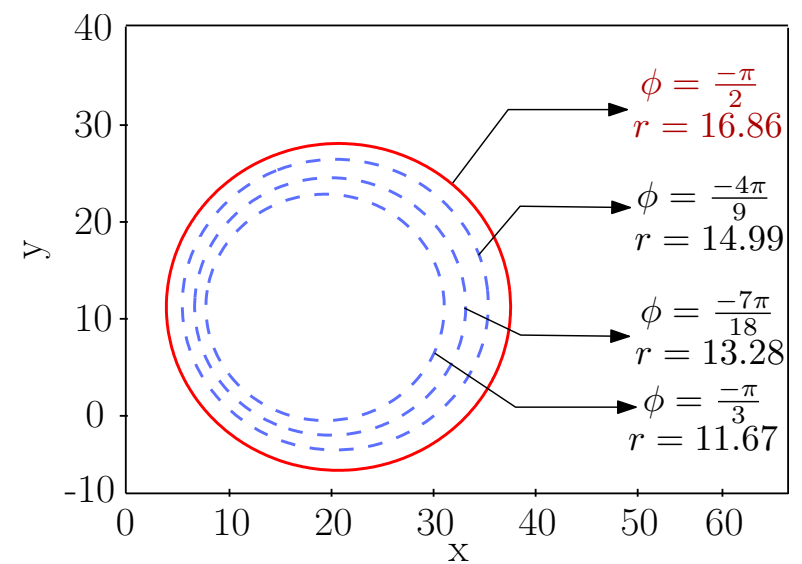

Fig. 2. The MSFC for $\phi=\left[-\frac{\pi}{2},-\frac{\pi}{3}\right]$ for the 3-RPR PM with geometrical parameters defined in table I.

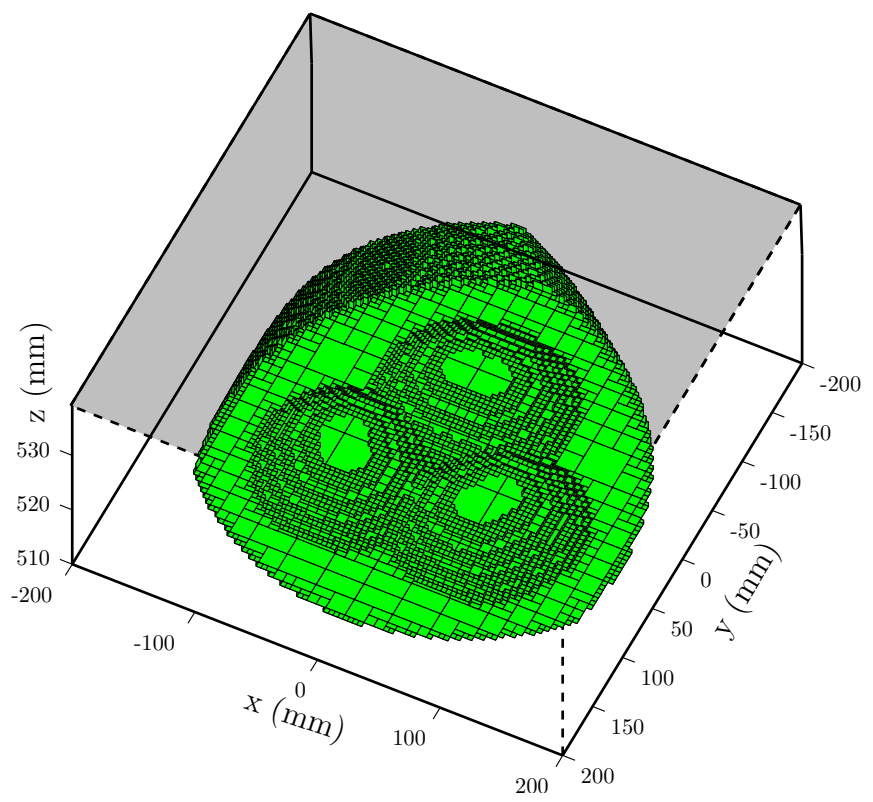

Fig. 3. The constant-orientation workspace of 6-UPS parallel robot with design properties as given in Table II, using interval analysis. section of $z=[510,540]$.

two new boxes where $\left[\mathbf{b}_{2}\right]$ contains the center of the MSFC, $C_{f}$. Finally, Fig. 2, obtained with Algorithm 4, depicts for obtained MSFC for several moving platform orientation, $\phi=\left[-\frac{\pi}{2},-\frac{\pi}{3}\right]$. Figure 2 shows that the radius $r$ of the MSFC is a maximum for $\phi=-\frac{\pi}{2}, r=16.86 \mathrm{~mm}$.

\section{B. Results Obtained for 6-U $\underline{P S}$ Planar Parallel Mechanism}

This section represents the results obtained from using the proposed algorithm to find the MSFS for a 6-UPS parallel robot with the design parameters given in Table II.

Figure 3 represents the constant-orientation workspace for $\theta=0, \phi=0, \psi=0$ and $z=[510,540]$, obtained with Algorithm 1. In turn, for the same set of orientations used for Fig. 3, Figs. 4(a) and 4(b) represent respectively the implicit and interval-based (Algorithm 2) representations of the singularity loci of the parallel robot under study. 
TABLE II

GEOMETRIC PARAMETERS OF THE 6-UPS PARALLEL ROBOT UNDER STUDY (ALL LENGTHS ARE GIVEN IN $\mathrm{mm}$ ).

\begin{tabular}{ccccccc}
\hline \hline$i$ & 1 & 2 & 3 & 4 & 5 & 6 \\
\hline$x_{a i}$ & 92.58 & 132.58 & 40.00 & -40.00 & -132.58 & -92.58 \\
$y_{a i}$ & 99.64 & 30.36 & -130.00 & -130.00 & 30.36 & 99.64 \\
$z_{a i}$ & 23.10 & 23.10 & 23.10 & 23.10 & 23.10 & 23.10 \\
$x_{b i}$ & 30.00 & 78.22 & 48.22 & -48.22 & -78.22 & -30.00 \\
$y_{b i}$ & 73.00 & -10.52 & -62.48 & -62.48 & -10.52 & 73.00 \\
$z_{b i}$ & -37.10 & -37.10 & -37.10 & -37.10 & -37.10 & -37.10 \\
$\rho_{i_{\min }}$ & 454.5 & 454.5 & 454.5 & 454.5 & 454.5 & 454.5 \\
$\rho_{i_{\max }}$ & 504.5 & 504.5 & 504.5 & 504.5 & 504.5 & 504.5 \\
\hline \hline
\end{tabular}

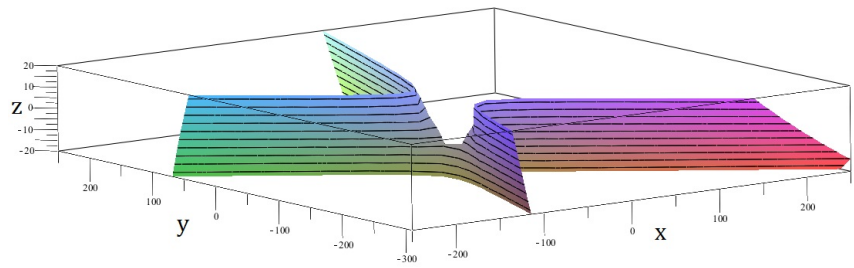

(a) Implicit representation of the singularity loci of a 6-UPS parallel robot

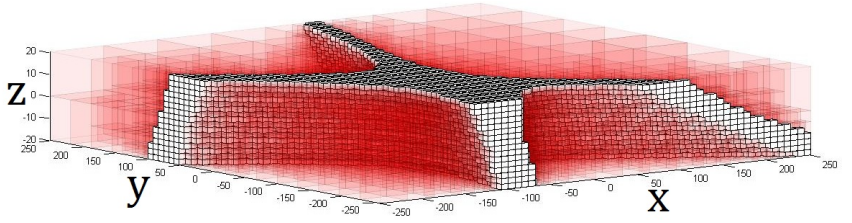

(b) Singularity loci surface of a 6-UPS parallel robot, obtained from Algorithm 1 . The set of red transparent boxes are outside of the singularity loci and white boxes lie on the singularity loci

Fig. 4. Singularity loci of the 6-UPS parallel robot with design parameters given in Table II: (a) implicitly depicted (b) interval-based

Moreover, as it can be observed in Fig. 5, the MSFS is tangent to both singularity locus and workspace boundaries. By inspection, it can be inferred that the obtained MSFS corresponds to the optimal inscribed sphere bounded by the workspace and the singularity locus.

For the sake of a better understanding, Fig. 6 represents a cross-sectional view of the result depicted in Fig. 5. It is worth mentioning that, in Fig. 6, the gray circles are crosssection views of the MSFS in different $x y$-planes. As it can be observed in Fig. 6, the gray circles are neither tangent to the workspace boundaries nor to the singularity locus. The latter statement is in accordance with the fact that the MSFS should absolutely be tangent to the constraints of the problem, since this takes place in a 3-dimensional space. Indeed, the set of points that are tangent to the workspace or singularity locus, are not lying in the prescribed crosssectional planes along the $z$-axis.

\section{CONCLUSiOns AND Future Works}

This paper presented four interval-based algorithms, Algorithms 1, 2, 3 and 4 , in order to obtain respectively the constant-orientation workspace, the singularity, the maximal singularity-free workspace for a given box and the maximal singularity-free workspace for the entire workspace. As

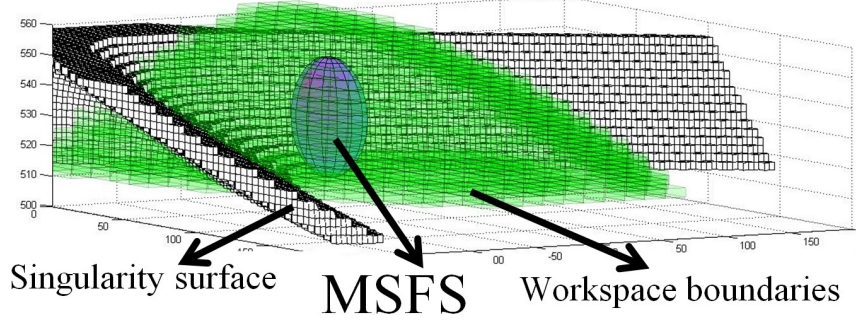

Fig. 5. The MSFS of a 6-UPS parallel robot for $\theta=0, \phi=0, \psi=$ 0 and $z=[510,550]$. The set of white boxes represents the singularity surface and green-transparent boxes are inside the constant-orientationworkspace. MSFS is obtained in such a way that it is tangent to both the singularity surface and the workspace.

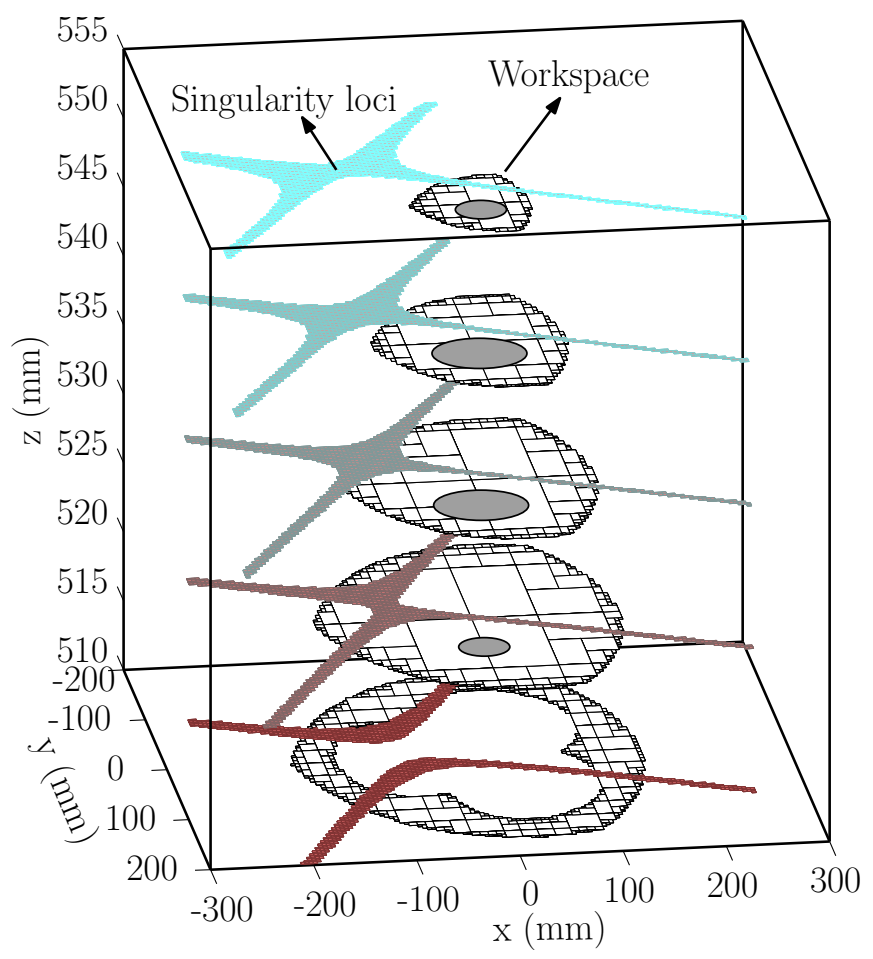

Fig. 6. Interval-based results for the MSFS for $\theta=0, \phi=0$, and $\psi=$ 0 . Only cross-sectional plans are considered to represent the constantorientation workspace and the singularity locus of the robot not to overload the figure. The gray circles represent sections of the MSFS.

case studies, the proposed algorithms were used to obtain Maximal Singularity-free Circle (MSFC) for a 3-DOF planar parallel robot i.e. 3-RPR and the Maximal singularity-free Sphere (MSFS) for a 6-DOF parallel robot i.e. 6-UPS MSSM Gough-Stewart platform. The proposed algorithms laid down the state-of-the-art for formulating the problem of finding the maximal singularity-free workspace of parallel robots. However, it could be readily extended to other robots, and open an avenue to find a systematic approach to do so. The results obtained from these algorithms revealed that the proposed algorithms are robust and could be also used for the optimum synthesis of the robots under study. Ongoing works include the extension of the proposed algorithms to the analysis of complex parallel robots and the dimension synthesis of parallel robots based on the algorithms presented 


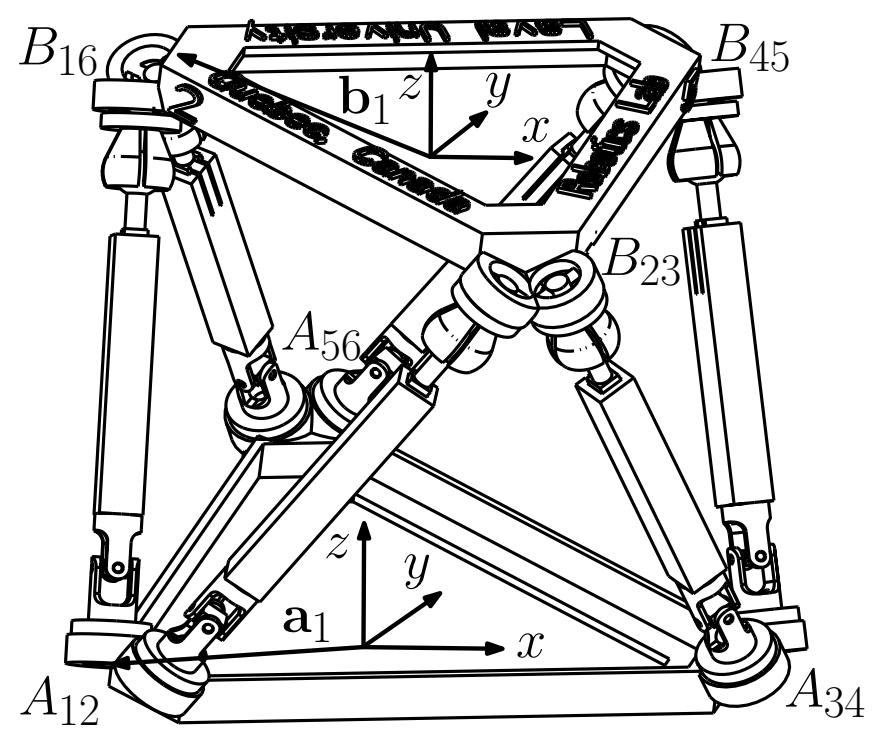

Fig. 7. The MSSM Gough-Stewart platform. The schematic is adapted from [40].

in this paper.

\section{APPENDIX}

\section{A. 6-UPS Parallel Mechanism}

In this section, three important kinematic properties, namely IKP, singularity analysis and workspace are broadly reviewed. The IKP pertains to finding the values of joint variables for a given position and orientation of the EndEffector (EE). Figure 7 represents a Minimal Simplified Symmetric Manipulator (MSSM) 6-UPS parallel robot. It should be noted that $\underline{\mathrm{P}}$ stands for an actuated prismatic joint. In order to clearly establish the notation used here (inspired from [38]), consider a fixed coordinate frame $R: O-x y z$ attached to the base platform and a moving coordinate frame $R^{\prime}: O^{\prime}-x^{\prime} y^{\prime} z^{\prime}$ attached to the EE. Moreover, the $i^{\text {th }}$ leg is attached to the base platform at point $A_{i}$ and to the EE at point $B_{i}$. Vectors $\mathbf{a}_{i}$ and $\mathbf{b}_{i}, i=1, \ldots, 6$, are the position vectors of point $A_{i}$ and $B_{i}$, expressed in frames $R$ and $R^{\prime}$ respectively. Furthermore, $\mathbf{Q}$ denotes the rotation matrix characterizing the orientation between frame $R$ and frame $R^{\prime} . \rho_{i}$ is the joint variable of the $i^{\text {th }}$ prismatic joint. The position vector of point $B_{i}$ expressed in the fixed frame $R$ can be written as:

$$
\left[\mathbf{b}_{i}\right]_{R}=[\mathbf{r}]_{R}+\mathbf{Q}\left[\mathbf{b}_{i}\right]_{R^{\prime}}, i=1, \ldots, 6
$$

where $[\mathbf{r}]_{R}=\left[x_{r}, y_{r}, z_{r}\right]^{T}$ stands for the position vector of point $O^{\prime}$ expressed in frame $R$ and the subscript $R$ indicates that the corresponding vector is expressed in frame $R$. Subtracting $\mathbf{a}_{i}$ from both sides of Eq. (7) leads to:

$$
\left[\mathbf{b}_{i}-\mathbf{a}_{i}\right]_{R}=[\mathbf{r}]_{R}+\mathbf{Q}\left[\mathbf{b}_{i}\right]_{R^{\prime}}-\left[\mathbf{a}_{i}\right]_{R} .
$$

The left-side of Eq. (8) is clearly the vector connecting point $A_{i}$ to point $B_{i}$, hence, by taking the Euclidean norm of each side, one can obtain the IKP of the $i^{\text {th }}$ limb as follows:

$$
\rho_{i}=\left\|\left[\mathbf{b}_{i}-\mathbf{a}_{i}\right]_{R}\right\|_{2}=\left\|[\mathbf{r}]_{R}+\mathbf{Q}\left[\mathbf{b}_{i}\right]_{R^{\prime}}-\left[\mathbf{a}_{i}\right]_{R}\right\|_{2},
$$

where $\|\cdot\|_{2}$ stands for the Euclidean norm. Therefore, for a given robot, the actuated variable $\rho_{i}$ can be directly computed for a given position and orientation of the EE.

In this paper, the actuation singularity, referred to as Type II [7] is more of concern, which occurs when the moving platform possesses certain DOF whereas the actuators are locked. As a necessary condition, the rank of the actuation system of parallel robot in a non-singular configuration should be equal to six. Once this rank decreases, an infinitesimal motion of the EE will occur and the platform will be uncontrollable. Upon resorting to screw theory [14] and [1], one can write the kinematical screw system, $\$_{i}$, for a 6-U $\underline{P S}$ limb as:

$$
\$_{\mathbf{i}}=\left[\begin{array}{cc}
\mathbf{e}_{1}^{T} & \left(\mathbf{a}_{i} \times \mathbf{e}_{1}\right)^{T} \\
\mathbf{e}_{2}^{T} & \left(\mathbf{a}_{i} \times \mathbf{e}_{1}\right)^{T} \\
\mathbf{0}^{T} & \mathbf{e}_{\rho_{i}}^{T} \\
\mathbf{e}_{1}^{T} & \left(\mathbf{b}_{i} \times \mathbf{e}_{1}\right)^{T} \\
\mathbf{e}_{2}^{T} & \left(\mathbf{b}_{i} \times \mathbf{e}_{2}\right)^{T} \\
\mathbf{e}_{3}^{T} & \left(\mathbf{b}_{i} \times \mathbf{e}_{3}\right)^{T}
\end{array}\right], \quad i=1, \ldots 6
$$

in which $\mathbf{e}_{\rho_{i}}$ is the unit vector of the direction of the $i^{\text {th }}$ prismatic joint. From Eq. (10), it can be concluded that, no constraint wrench is imposed by the limb to the EE, therefore the robot under study has 6-DOF. Furthermore, the forward Jacobian matrix of 6-UPS robot takes the form:

$$
\mathbf{J}=\left[\begin{array}{cc}
\mathbf{e}_{\rho_{1}}^{T} & \left(\mathbf{b}_{1} \times \mathbf{e}_{\rho_{1}}\right)^{T} \\
\mathbf{e}_{\rho_{2}}^{T} & \left(\mathbf{b}_{2} \times \mathbf{e}_{\rho_{\mathbf{2}}}\right)^{T} \\
\mathbf{e}_{\rho_{\mathbf{3}}}^{T^{2}} & \left(\mathbf{b}_{3} \times \mathbf{e}_{\rho_{\mathbf{3}}}\right)^{T} \\
\mathbf{e}_{\rho_{\mathbf{4}}}^{T} & \left(\mathbf{b}_{4} \times \mathbf{e}_{\rho_{\mathbf{4}}}\right)^{T} \\
\mathbf{e}_{\rho_{\mathbf{5}}}^{T} & \left(\mathbf{b}_{5} \times \mathbf{e}_{\rho_{\mathbf{5}}}\right)^{T} \\
\mathbf{e}_{\rho_{\mathbf{6}}}^{T} & \left(\mathbf{b}_{6} \times \mathbf{e}_{\rho_{\mathbf{6}}}\right)^{T}
\end{array}\right]
$$

Each row of the Jacobian matrix is a Plücker line and corresponds to a screw reciprocal to all the passive twists of the corresponding limb, but not to the actuated twist. $\operatorname{det}(\mathbf{J})=0$ represents the singularity locus of the robot.

The workspace of a robot consists in the set of Cartesian points that can be reached by the EE of the manipulator. The solution of the IKP can be used to obtain the workspace of the robot for a given mechanical stroke associated to each limb, $\rho_{\min }<\rho_{i}<\rho_{\max } . \rho_{\min }$ and $\rho_{\max }$ are the lower bound and the upper bound of the actuated prismatic joints, respectively. Hence, each point of the Cartesian space that satisfies Eq. (9) for the given stroke, belongs to the workspace of the manipulator. Furthermore, the workspace of a PM can be resorted as a geometrical reasoning. For instance, the workspace of a 6-UPS parallel robot is the common area of the intersection of six inner and six outer spheres, known as vertex space. The inner spheres correspond to the lower limit of the actuated prismatic joints and the outer ones correspond to the upper limits of the actuated prismatic joints. 


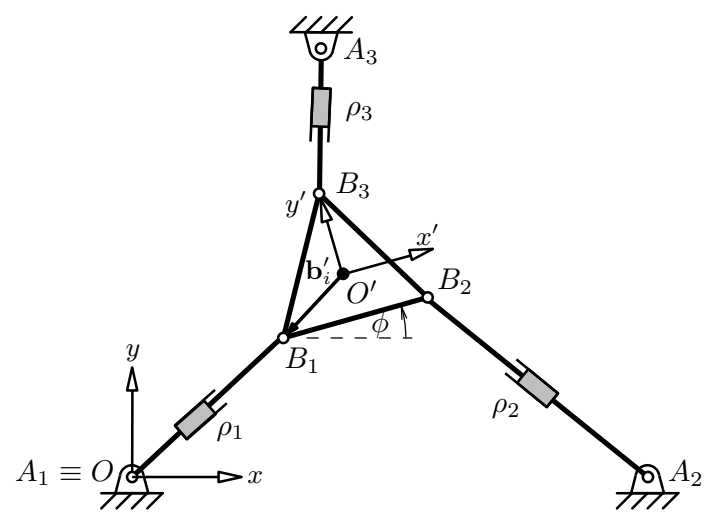

Fig. 8. A schematic representation for a 3-RPR PM [41].

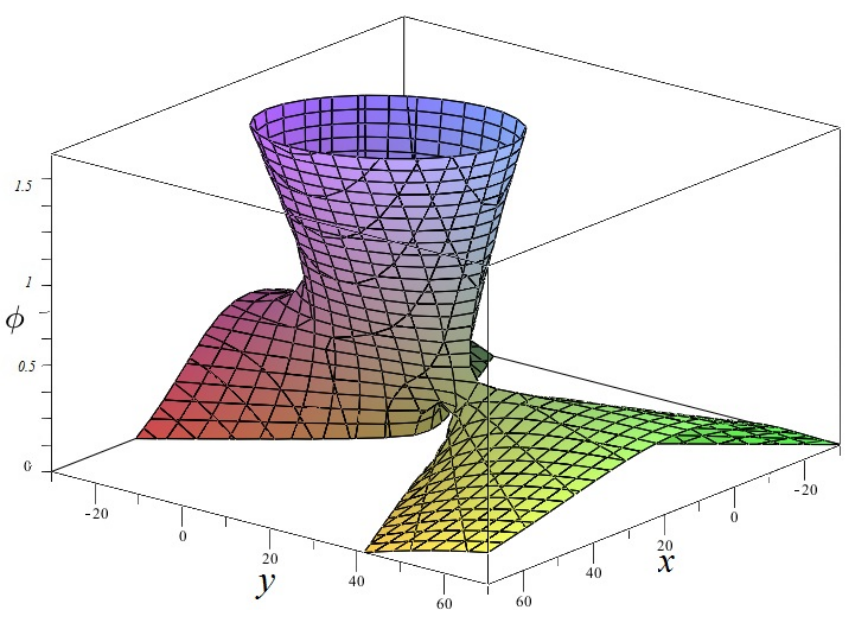

Fig. 9. Three dimensional singularity locus of a 3-RPR PM for $\phi=\left[0, \frac{\pi}{2}\right]$.

\section{B. 3-RPR Planar Parallel Mechanism}

Figure 8 illustrates the schematic representation of a 3RPR PPM. As depicted in Fig. 8, a planar 3-RPR PM with actuated prismatic joints consists of a fixed triangular $\left(\Delta_{A_{1} A_{2} A_{3}}\right)$ and a mobile triangular platform $\left(\Delta_{B_{1} B_{2} B_{3}}\right)$. The passive revolute joints, located at $A_{i}$ and $B_{i}$, are connected by the prismatic actuator of variable length $\rho_{i}, i=1,2,3$. The IKP of a $3-\mathrm{RPR}$ is similar to Eq. (9) with $i=1,2,3$.

The first-order kinematic relation, coming from the differentiation of the IKP with respect to time, of a 3-RPR PM in a matrix form, called the Jacobian, can be expressed as:

$$
\mathbf{J}=\left[\begin{array}{lll}
n_{1 x} & n_{1 y} & \left(\mathbf{b}_{1} \times \mathbf{n}_{1}\right) \cdot \mathbf{k} \\
n_{2 x} & n_{2 y} & \left(\mathbf{b}_{2} \times \mathbf{n}_{2}\right) \cdot \mathbf{k} \\
n_{3 x} & n_{3 y} & \left(\mathbf{b}_{3} \times \mathbf{n}_{3}\right) \cdot \mathbf{k}
\end{array}\right]
$$

In the above, $\mathbf{k}$ is the unit vector along $z$-axis, $\mathbf{b}_{i}, i=$ $1,2,3$, is the position vector of point $B_{i}$ expressed in the fixed frame and the unit vector along the $i^{\text {th }}$ prismatic joint direction is denoted by $\mathbf{n}_{i}=\left[n_{i_{x}}, n_{i_{y}}, 0\right]^{T}$.

More information concerning the kinematic properties of 3-RPR PMs can be found in [41]. In short, the singularity, or more precisely the Type II singularity [7], are those pose, i.e., position and orientation $(x, y, \phi)$, for which the determinant of $\mathbf{J}$ vanishes:

$$
D(x, y, \phi)=\operatorname{det}(\mathbf{J})=0
$$

From [19], [42], that the singularity curves of a $3-R \underline{P R}$ fall into three types for different orientations of the moving platform: (1) a hyperbola (2) a parabola or (3) an ellipse (ellipse may degenerate into a circle). The latter can be also inferred by simple observation of Fig. 9.

\section{REFERENCES}

[1] X. Kong and C. Gosselin, Type Synthesis of Parallel Mechanisms. Springer, Heidelberg, 2007, vol. 33.

[2] B. Li, Y. Cao, Q. Zhang, and Z. Huang, "Position-singularity analysis of a special class of the stewart parallel mechanisms with two dissimilar semi-symmetrical hexagons," Robotica, vol. 31, pp. 123136, 12013.

[3] X.-J. Liu, J. Wang, F. Gao, and L.-P. Wang, "Mechanism design of a simplified 6-dof 6-rus parallel manipulator," Robotica, vol. 20, pp. 81-91, 12002.

[4] T. Li and S. Payandeh, "Design of spherical parallel mechanisms for application to laparoscopic surgery," Robotica, vol. 20, pp. 133-138, 32002.

[5] G. Bhutani and T. A. Dwarakanath, "Practical feasibility of a highprecision 3-upu parallel mechanism," Robotica, vol. FirstView, pp. 1$15,82013$.

[6] "Parallelmic, http://www.parallemic.org/."

[7] C. Gosselin and J. Angeles, "Singularity Analysis of Closed-Loop Kinematic Chains," IEEE Transactions on Robotics and Automation, vol. 6, no. 3, pp. 281-290, 1990.

[8] M. Tale Masouleh and C. Gosselin, "Determination of Singularityfree Zones in the Workspace of Planar 3-PRR Parallel Mechanisms,' Journal of Mechanical Design, vol. 129, p. 649, 2007.

[9] S. Bhattacharya, H. Hatwal, and A. Ghosh, "On the optimum design of stewart platform type parallel manipulators," Robotica, vol. 13, pp. 133-140, 31995

[10] J. P. Merlet, Parallel Robots. Springer, 2006

[11] P. Voglewede and I. Ebert-Uphoff, "Overarching Framework for Measuring Closeness to Singularities of Parallel Manipulators," IEEE Transactions on Robotics, vol. 21, no. 6, pp. 1037-1045, 2005.

[12] J. P. Merlet and P. Donelan, "On the Regularity of the Inverse Jacobian of Parallel Robots," Advances in Robot Kinematics, pp. 41-48, 2006.

[13] M. Conconi and M. Carricato, "A New Assessment of Singularities of Parallel Kinematic Chains," IEEE Transactions on Robotics, vol. 25, no. 4, pp. 757-770, 2009.

[14] I. A. Bonev, D. Zlatanov, and C. M. Gosselin, "Singularity Analysis of 3-DOF Planar Parallel Mechanisms Via Screw Theory," Journal of Mechanical Design, vol. 125, p. 573, 2003.

[15] Y. Yang and J. O'Brien, “A Case Study of Planar 3-RPR Parallel Robot Singularity Free Workspace Design," in International Conference on Mechatronics and Automation (ICMA). IEEE, 2007, pp. 1834-1838.

[16] H. Li, C. Gosselin, and M. Richard, "Determination of Maxima Singularity-free Zones in the Workspace of Planar Three-degreeof-freedom Parallel Mechanisms," Mechanism and Machine Theory, vol. 41, no. 10, pp. 1157-1167, 2006.

[17] Q. Jiang and G. C.M., "Geometric Synthesis of Planar 3-RPR Parallel Mechanisms for Singularity-free workspace," Transactions of the Canadian Society for Mechanical Engineering, vol. 33, no. 4, pp. 667 678, 2009.

[18] Q. Jiang and C. Gosselin, "The Maximal Singularity-Free Workspace of Planar 3-RPR Parallel Mechanisms," in Proceedings of the 2006 International Conference on Mechatronics and Automation. IEEE, 2006, pp. 142-146.

[19] Jiang and Gosselin, "Geometric Optimization of Planar 3-RPR Parallel Mechanisms," Transactions of the Canadian Society for Mechanical Engineering, vol. 31, no. 4, pp. 457-468, 2007.

[20] G. Abbasnejad, H. Daniali, and S. Kazemi, "A New Approach to Determine the Maximal Singularity-free Zone of 3-RPR Planar Parallel Manipulator," Robotica, vol. 1, no. 1, pp. 1-8.

[21] Q. Jiang, "Singularity-free workspace analysis and geometric optimization of parallel mechanisms," Ph.D. dissertation, Université Laval, 2008., 2008. 
[22] J.-P. Merlet, D. Daney et al., "A formal-numerical approach to determine the presence of singularity within the workspace of a parallel robot." in In 2nd Workshop on Computational Kinematics, 2001, pp. $167-176$.

[23] M. Gallant and R. Boudreau, "The synthesis of planar parallel manipulators with prismatic joints for an optimal, singularity-free workspace," Journal of Robotic Systems, vol. 19, no. 1, pp. 13-24, 2002.

[24] H. Li, C. Gosselin, and R. M.J, "Determination of the Maximal Singularity-free Zones in the Six-dimensional Workspace of the General Gough-Stewart Platform," Mechanism and machine theory, vol. 42, no. 4, pp. 497-511, 2007.

[25] R. E. Moore and F. Bierbaum, Methods and Applications of Interval Analysis. Society for Industrial Mathematics, 1979, vol. 2.

[26] F. Abdallah, A. Gning, and P. Bonnifait, "Box particle filtering for nonlinear state estimation using interval analysis," Automatica, vol. 44, no. 3, pp. 807-815, 2008.

[27] P. Dwyer, "Computation with approximate numbers," pp. 11-34, 1951.

[28] T. Sungana, "Theory of Interval Algebra and Application to Numerical Analysis," pp. 29-46, 1958.

[29] M. Warmus, "Calculus of approximations," Bulletin de lAcademie Polonaise de Sciences, vol. 4, no. 5, pp. 253-257, 1956.

[30] J. Wilkinson, "Turings work at the national physical laboratory and the construction of pilot ace, deuce, and ace," Metropolis et al.[MHR80], pp. 101-114, 1980.

[31] R. E. Moore, "Interval analysis. series in automatic computation," Englewood Cliff: NJ Prentice-Hall, 1966.

[32] E. Hansen and G. Walster, Global Optimization Using Interval Analysis: Revised and Expanded. CRC, 2003, vol. 264.

[33] J. P. Merlet, "Solving the Forward Kinematics of a Gough-type Parallel Manipulator with Interval Analysis," The International Journal of robotics research, vol. 23, no. 3, pp. 221-235, 2004.

[34] F. Hao and J. P. Merlet, "Multi-criteria Optimal Design of Parallel Manipulators Based on Interval Analysis," Mechanism and Machine Theory, vol. 40, no. 2, pp. 157-171, 2005.

[35] J. Merlet, "Interval Analysis and Robotics," Robotics Research, pp. 147-156, 2011.

[36] D. Chablat, P. Wenger, F. Majou, and J. P. Merlet, "An interval analysis based study for the design and the comparison of three-degrees-offreedom parallel kinematic machines," The International Journal of Robotics Research, vol. 23, no. 6, pp. 615-624, 2004.

[37] D. Oetomo, D. Daney, B. Shirinzadeh, and J. P. Merlet, "Certified Workspace Analysis of 3RRR Planar Parallel Flexure Mechanism," in IEEE International Conference on Robotics and Automation (ICRA). IEEE, 2008, pp. 3838-3843.

[38] C. Gosselin, "Determination of the Workspace of 6-DOF Parallel Manipulators," ASME Journal of Mechanical Design, vol. 112, no. 3, pp. 331-336, 1990.

[39] M. Saadatzi, M. Tale Masouleh, and H. Taghirad, "Workspace Analysis of 5-PRUR Parallel Mechanisms (3T2R)," Robotics and ComputerIntegrated Manufacturing, 2012.

[40] Laboratoire de Robotique de L'Université Laval. [Online]. Available: http://www.robot.gmc.ulaval.ca

[41] I. A. Bonev, "Geometric Analysis of Parallel Mechanisms," Ph.D. dissertation, Laval University, Quebec, QC, Canada, October 2002.

[42] J. Sefrioui and C. Gosselin, "On the Quadratic Nature of the Singularity Curves of Planar Three-Degree-Of-Freedom Parallel Manipulators," Mechanism and Machine Theory, vol. 30, no. 4, pp. 553-551, 1995. 